\title{
Tendencias de mortalidad y años potenciales de vida perdidos por cáncer de ovario en México, 2000-2014
}

Juan Jesús Sánchez-Barriga

Secretaria de Salud, Dirección General de Epidemiología, Dirección de Investigación Operativa en Epidemiología, Ciudad de México, México

\section{Resumen}

Introducción: En México, el cáncer de ovario representa $5.3 \%$ de los diagnósticos de cáncer en todos los grupos de edad y 21 \% de los cánceres ginecológicos; en las últimas tres décadas ha tenido un aumento constante. Objetivo: Determinar la tendencia de la mortalidad por cáncer de ovario y los años potenciales de vida perdidos (APVP) por estado y región socioeconómica de México entre 2000 y 2014. Método: Se obtuvieron los registros de cáncer de ovario del Instituto Nacional de Estadística y Geografía. Se identificaron los códigos de la CIE-10 correspondientes a la causa básica de defunción por cáncer de ovario. Se calcularon las tasas de mortalidad y las tasas de APVP a nivel nacional, por estado y región socioeconómica. Resultados: Entre 2000 y 2014, las tasas ajustadas por edad por 100000 mujeres se incrementaron de 3.3 a 4.1. Las mayores tasas de mortalidad se identificaron en la región 7, en Chihuahua, Baja California Sur, Colima, Quintana Roo, Zacatecas, Sonora, Coahuila, Aguascalientes, Querétaro. La mayor tasa de APVP por cáncer de ovario se registró en las regiones 7, 5 y 6, en Nayarit, Baja California Sur, Zacatecas, Colima, Tlaxcala, Oaxaca, Quintana Roo, Coahuila, Aguascalientes y Querétaro. Conclusiones: La región socioeconómica 7 de México presentó las mayores tasas de APVP y de mortalidad por cáncer de ovario.

PALABRAS CLAVE: Cáncer de ovario. Mortalidad. Años potenciales de vida perdidos.

\begin{abstract}
Introduction: In Mexico, ovarian cancer accounting for $5.3 \%$ of cancer diagnoses in all age groups and $21 \%$ of gynecological cancers and it has had a steady increase in the last three decades. Objective: To determine mortality trends from ovarian cancer and potential years of life lost (PYLL) by state and socioeconomic region of Mexico between 2000 and 2014. Method: Records of ovarian cancer were obtained from the National Institute of Statistics and Geography. ICD-10 codes corresponding to ovarian cancer as the basic cause of death were identified. Mortality rates and YPLL rates were calculated by nationwide, states and socioeconomic region. Results: Between 2000 and 2014, age-adjusted rates per 100,000 women increased from 3.3 to 4.1. The highest mortality rates were identified in region 7, in Chihuahua, Baja California Sur, Colima, Quintana Roo, Zacatecas, Sonora, Coahuila, Aguascalientes and Queretaro. The highest rates of PYLL due to ovarian cancer were recorded in regions 7, 5 and 6, Nayarit, Baja California Sur, Zacatecas, Colima, Tlaxcala, Oaxaca, Quintana Roo, Coahuila, Aguascalientes and Queretaro. Conclusions: Mexico's socioeconomic region 7 had the highest rates of PYLL and mortality from ovarian cancer.
\end{abstract}

KEY WORDS: Ovarian cancer. Mortality. Potential years of life lost.

Fecha de recepción: 26-07-2017

Fecha de aceptación: 18-01-2018

DOI://dx.doi.org/10.24875/GMM.18003606
Gac Med Mex. 2018;154:438-447

Disponible en PubMed www.gacetamedicademexico.com 


\section{INTRODUCCIÓN}

El cáncer de ovario (CO) es la séptima neoplasia más frecuente en las mujeres y la décimo octavo más frecuente en el mundo. Aproximadamente 239000 casos se registraron en 2012, que representaron casi $4 \%$ de todos los nuevos casos de cáncer en mujeres ( $2 \%$ en total). Las tasas de incidencia por CO son mayores en los países de ingresos altos que en los de ingresos medios y bajos. En Europa Central y Oriental, en 2012, la tasa de incidencia estandarizada por edad fue de 11 por 100000 mujeres y en algunas partes de África fue menor de 5. En el Reino Unido fueron de 11.7 y en Estados Unidos de 8, en Brasil de 5.2 y en China de 4.1.

Durante las dos últimas décadas, las tasas de mortalidad por $\mathrm{CO}$ han presentado una tendencia a equilibrarse o disminuir en varios países europeos y de Norteamérica de altos ingresos, donde eran altas. El avance en el diagnóstico y tratamiento del $\mathrm{CO}$, así como el uso de anticonceptivos orales pudieron haber influido, particularmente en los países de altos ingresos. Existen diferencias persistentes y sustanciales en los patrones y tendencias del $\mathrm{CO}$ en el mundo. En 2012, en el mundo, el país con la mayor mortalidad fue Lituania, con una tasa de mortalidad de 7.1 por 100000 mujeres ajustada por edad con la población mundial; el país con la menor tasa de mortalidad fue la República de Corea con 2.2. En ese mismo año, en Europa, los países con las mayores tasas de mortalidad por CO fueron Lituania (7.1), Irlanda (7.09) y Letonia (6.64) y los países con menor mortalidad fueron España (3.69), Grecia (3.5) y Portugal (3.03). En la región de Asia, Australia y Oceanía los países con mayor mortalidad en el 2011 fueron Nueva Zelanda (4.93) y Australia (4.27) y los que tuvieron menor mortalidad fueron Hong Kong (2.46) y la República de Corea (2.26). En la región de las Américas, los países con mayor mortalidad en 2011 fueron Canadá (4.95) y en 2012, Estados Unidos (4.85); los que tuvieron menor mortalidad en este continente en 2012 fueron Cuba (3.07) y Brasil (2.8). ${ }^{2}$

En México, el CO representa $5.3 \%$ de los diagnósticos de cáncer en todos los grupos de edad y $21 \%$ de los cánceres ginecológicos. En 2004 se registraron 473417 defunciones, de las que los tumores malignos fueron la tercera causa de muerte con 31443 decesos; $4.6 \%$ de las defunciones del total de la población femenina fue por $\mathrm{CO}^{3} \mathrm{Se}$ ha observado incremento sostenido de la mortalidad por $\mathrm{CO}$ de $1.4 \%$ anual de 1980 a 2011. En comparación con
Canadá, Estados Unidos, Argentina y Uruguay, México presenta una tasa intermedia de mortalidad por cáncer de ovario. ${ }^{4}$

Los objetivos de este estudio fueron determinar las tendencias de mortalidad y los APVP por CO por estado y región socioeconómica de México en el periodo 2000-2014.

\section{Métodos}

Se empleó un diseño de estudio de tendencias de mortalidad. Los registros de mortalidad asociados con el CO de 2000-2014 se obtuvieron del Instituto Nacional de Estadística y Geografía (INEGI). ${ }^{5}$ Esta información es recolectada de los certificados de defunción emitidos en el país.

Se incluyeron todos los registros individuales de mortalidad en los cuales la causa de muerte fue el CO. Se identificaron los códigos de la Décima Edición de la Clasificación Internacional de Enfermedades que corresponden al $\mathrm{CO}$ como causa de muerte (C56). ${ }^{6}$

Se obtuvieron las tasas de mortalidad a nivel nacional, crudas y ajustadas por edad, por 100000 mujeres, tomando como población estándar a la población mundial. ${ }^{7,8}$ También se obtuvieron las tasas de mortalidad ajustadas por edad por 100000 mujeres de cada estado de residencia y de cada una de las siete regiones socioeconómicas establecidas por el INEGI (Tabla 1). ${ }^{9}$ La población que se utilizó para el ajuste de tasas fue la nacional, estimada por el Consejo Nacional de Población para los años 2000- 2014. ${ }^{10}$

El cambio anual medio se calculó mediante el análisis de regresión lineal simple. Mediante este análisis se determinó en el periodo de estudio si existió disminución o aumento de la tasa de mortalidad por $\mathrm{CO}$, tanto en las tasas brutas como en las tasas de mortalidad ajustadas por edad con la población mundial por 100000 mujeres. ${ }^{11}$

También se determinaron las tasas de APVP por 100000 mujeres ajustadas por edad según estado de residencia y región socioeconómica. Se utilizaron grupos de edad quinquenales; los límites de edad que se utilizaron para el cálculo fueron los siguientes: límite inferior fue 1 año y el superior, 70 años para el cálculo de las tasas de APVP, que se expresaron por 100000 mujeres. ${ }^{12,13}$ Las tasas de APVP ajustadas por edad de se calcularon separando los años perdidos en sus respectivos grupos de edad, con base en la edad del individuo; las tasas específicas por edad se calcularon con base en el grupo de edad de la población. Estas 
tasas de APVP específicas por edad se ponderaron empleando la población nacional como la población estándar y se calculó una tasa única. La tasa calculada de APVP se obtuvo con la siguiente fórmula:13

\section{Tasas de APVP ajustadas por edad =}

$$
\sum_{i=1}^{69} a i\left(\frac{d i}{p i}\right)\left(\frac{P i r}{N r}\right) \times 100000
$$

Tabla 1. Regiones socioeconómicas de México

\begin{tabular}{ll}
\hline $\begin{array}{l}\text { Regiones } \\
\text { socioeconómicas }\end{array}$ & Estados \\
\hline 1 & Chiapas, Guerrero, Oaxaca \\
2 & $\begin{array}{l}\text { Campeche, Hidalgo, Puebla, San Luis } \\
\text { Potosí, Tabasco, Veracruz }\end{array}$ \\
3 & $\begin{array}{l}\text { Durango, Guanajuato, Michoacán, Tlaxcala, } \\
\text { Zacatecas }\end{array}$ \\
4 & $\begin{array}{l}\text { Colima, Estado de México, Morelos, Nayarit, } \\
\text { Querétaro, Quintana Roo, Sinaloa, Yucatán }\end{array}$ \\
5 & $\begin{array}{l}\text { Baja California, Baja California Sur, } \\
\text { Chihuahua, Sonora, Tamaulipas }\end{array}$ \\
6 & $\begin{array}{l}\text { Aguascalientes, Coahuila, Jalisco, Nuevo } \\
\text { León }\end{array}$ \\
7 & Ciudad de México \\
\hline Fuente: Instituto Nacional de Estadística y Geografía.
\end{tabular}

Donde

$p i=$ número de personas de edad $i$ en la población actual.

Pir $=$ número de personas de edad $i$ en la población de referencia.

$\mathrm{Nr}=$ número de personas entre 1 y 70 años en la población de referencia.

$d i=$ número de muertes entre edades $i e+1$.

$a i=$ años de vida restante hasta la edad de los 70 años, cuando mueren entre las edades $i \mathrm{e}$ $i+1=70-(i+0.5)$.

Se definieron las siete regiones socioeconómicas por el INEGI de acuerdo con el XII Censo General de Población y Vivienda, presentando las diferencias observadas en las condiciones sociales y económicas de la población en México. Los 32 estados se agrupan en las siete regiones socioeconómicas de acuerdo con los siguientes indicadores de bienestar: educación, ocupación, salud, vivienda y empleo. Los estados con características similares se clasificaron en una misma región y cada región es diferente de la otra. De acuerdo con los indicadores utilizados, las condiciones socioeconómicas tuvieron un rango en orden ascendente desde la región 1, la menos favorable, hasta la región 7 , la más favorable. El objetivo de la metodología empleada para establecer las regiones fue la

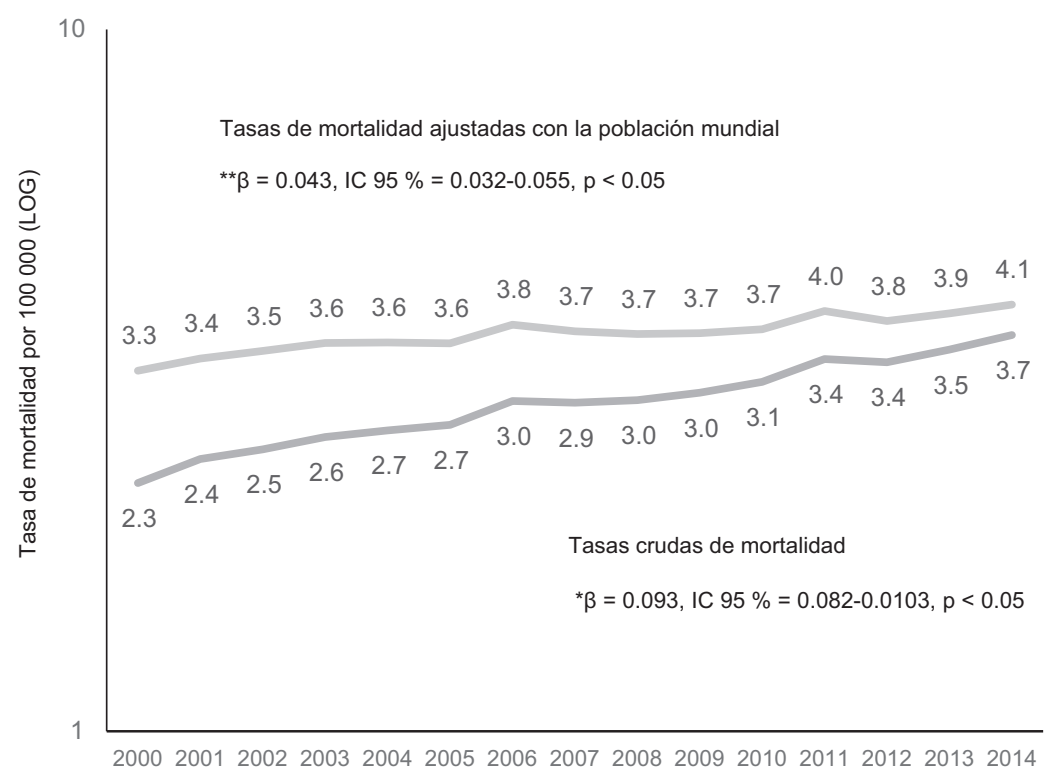

Año de muerte

Figura 1. Mortalidad por cáncer de ovario en México, 2000-2014. Tasa bruta de mortalidad por 100000 mujeres. Tasa ajustada por edad por método directo, estandarizada con la población mundial por 100000 mujeres. Fuente: Análisis por el autor de los datos obtenidos del Instituto Nacional de Estadística y Geografía, y del Consejo Nacional de Población: estimaciones de la población, periodo $1990-2010$ y proyecciones de población para 2010-2030. *Cambio anual medio por 100000 mujeres/año e IC 95 \% y valor de p para las tasas crudas de mortalidad en el periodo 2000-2014. ${ }^{* *}$ Cambio anual medio por 100000 mujeres/año e IC $95 \%$ y valor de p, para las tasas estandarizadas de mortalidad en el periodo 2000-2014. 


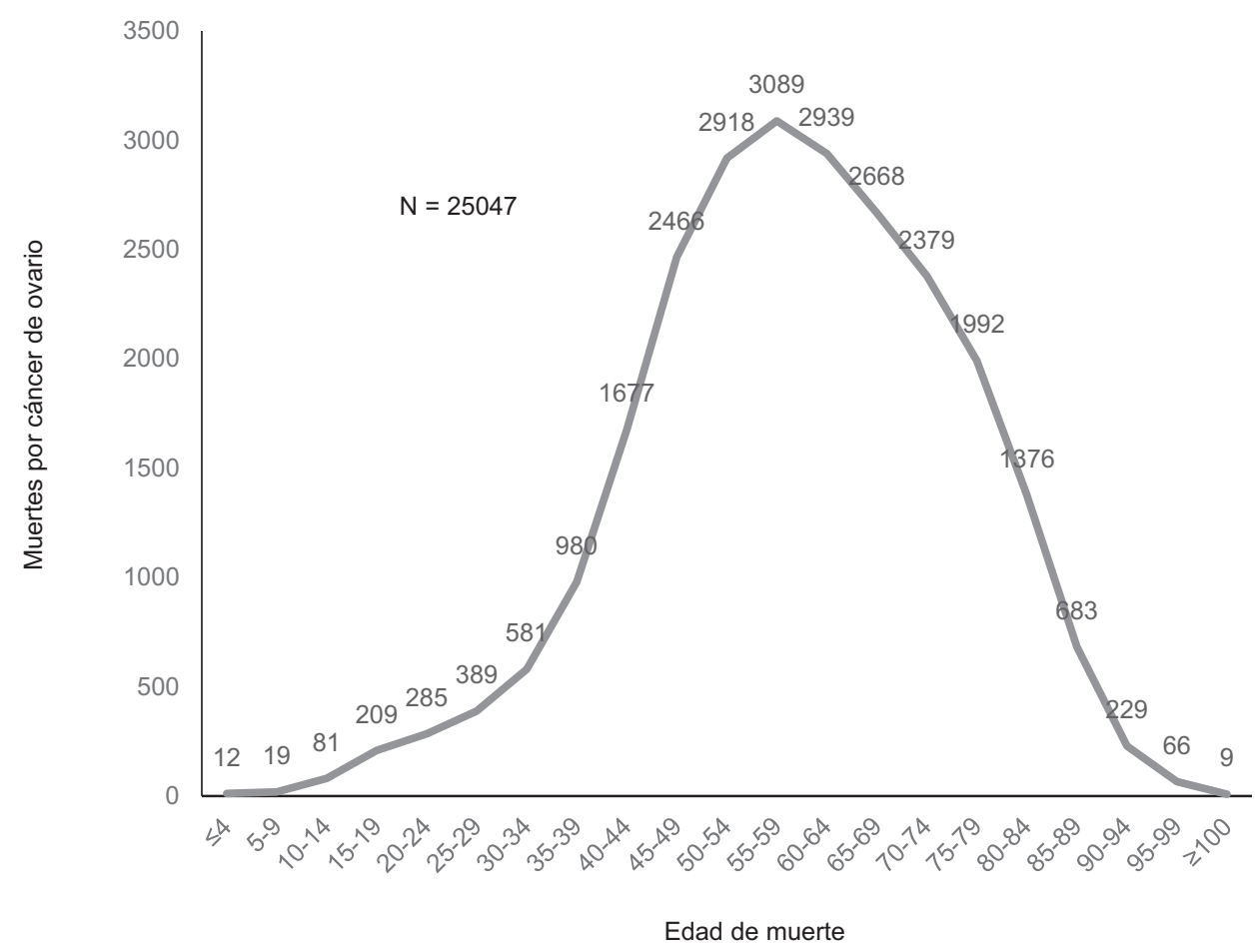

Figura 2. Mortalidad por cáncer de ovario por grupo de edad, México, 2000-2014. Análisis de la base de datos de mortalidad del Instituto Nacional de Estadística y Geografía.

formación de estratos con una variación mínima y de esta manera, apegados a un criterio de similitud establecido, agrupar los elementos de mayor semejanza permitiendo la distinción entre una región y otra. Entre las técnicas utilizadas estuvieron las distancias de Mahalonobis y una combinación de análisis factorial y el algoritmo K-medias. ${ }^{9}$

Los registros se manejaron con el programa Access 2013. Para el cálculo de las tasas de mortalidad ajustadas por edad y las tasas de APVP se utilizó el programa Epidat versión 3.1.14

\section{Resultados}

En México, en el periodo 2000-2014 fallecieron 498071 mujeres por neoplasias malignas y de estas, 25047 murieron por CO (5.02\% del total). En el 2000 se presentaron 1157 muertes y en 2014, 2249 por esta neoplasia. En el periodo de estudio, la tasa de mortalidad estandarizada por edad con la población mundial por 100000 mujeres se incrementó de 3.3 a 4.1, con un porcentaje de cambio de $24.2 \%$ y un cambio anual medio de 0.043, IC $95 \%=0.32-0.55$ (Figura 1).

A mayor edad, la mortalidad por $\mathrm{CO}$ se incrementó. Las mujeres con edad entre 55 y 59 años presentaron la mayor mortalidad (3089 casos, $12.3 \%$ ) (Figura 2).
Las tendencias de mortalidad especificas por grupo de edad se incrementaron entre 2000 y 2014, excepto en el grupo < 30 años. El grupo que presentó el mayor incremento en la mortalidad fue el de 51 a 60 años (datos no mostrados).

En el periodo, 31 estados presentaron un incremento en las tendencias de mortalidad por $\mathrm{CO}$, el único que no presentó cambios fue Nayarit. Los estados con las mayores tasas de mortalidad fueron Chihuahua en el año 2000 (3.8, IC $95 \%=2.75-4.85)$, Baja California Sur en 2001, 2002, 2007 y 2009 (5.36, IC $95 \%=2.14-8.58 ; 4.27$, IC $95 \%=1.3-7.25 ; 6.03$, IC $95 \%=2.8-9.27$; 4.44, IC $95 \%=1.76-7.11$, respectivamente); Colima en 2003, 2005, 2011, 2012 (4.75, IC $95 \%=2.25-7.24 ; 5.28$, IC $95 \%=2.69-7.87 ; 4.46$, IC $95 \%=2.26-6.72 ; 4.36$, IC $95 \%=2.15-6.57$, respectivamente); Quintana Roo en 2004 (4.26, IC $95 \%$ = 1.63-6.89); Zacatecas en 2006 (4.39, IC $95 \%=$ 2.85-5.9); Sonora en 2008 (3.98, IC $95 \%=2.88$ 5.07); Coahuila en 2010 (4.41, IC $95 \%=3.29-5.53)$; Aguascalientes en $2013(4.6$, IC $95 \%=2.86-6.34)$ y Querétaro en 2014 (5.2, IC $95 \%$ = 3.72-6.68) (Tabla 2). Los estados que presentaron la menor tasa de mortalidad fueron Colima en 2000 y 2002 (1.01, IC $95 \%$ $=-0.14-2.16$ y 1.34 , IC $95 \%=0.02-2.67$, respectivamente); Nayarit en 2001, 2007, 2009 y 2013 (1.14, 


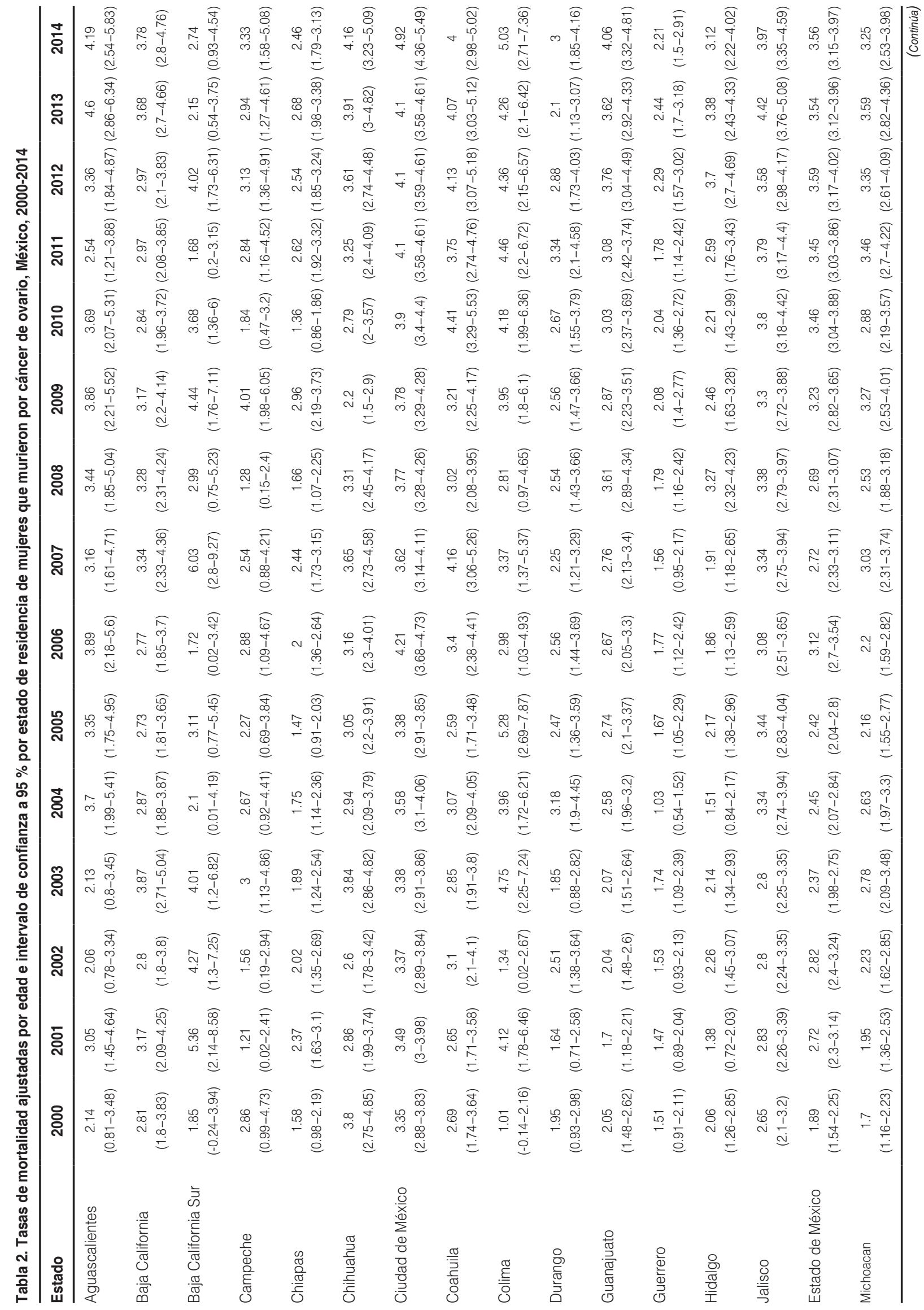




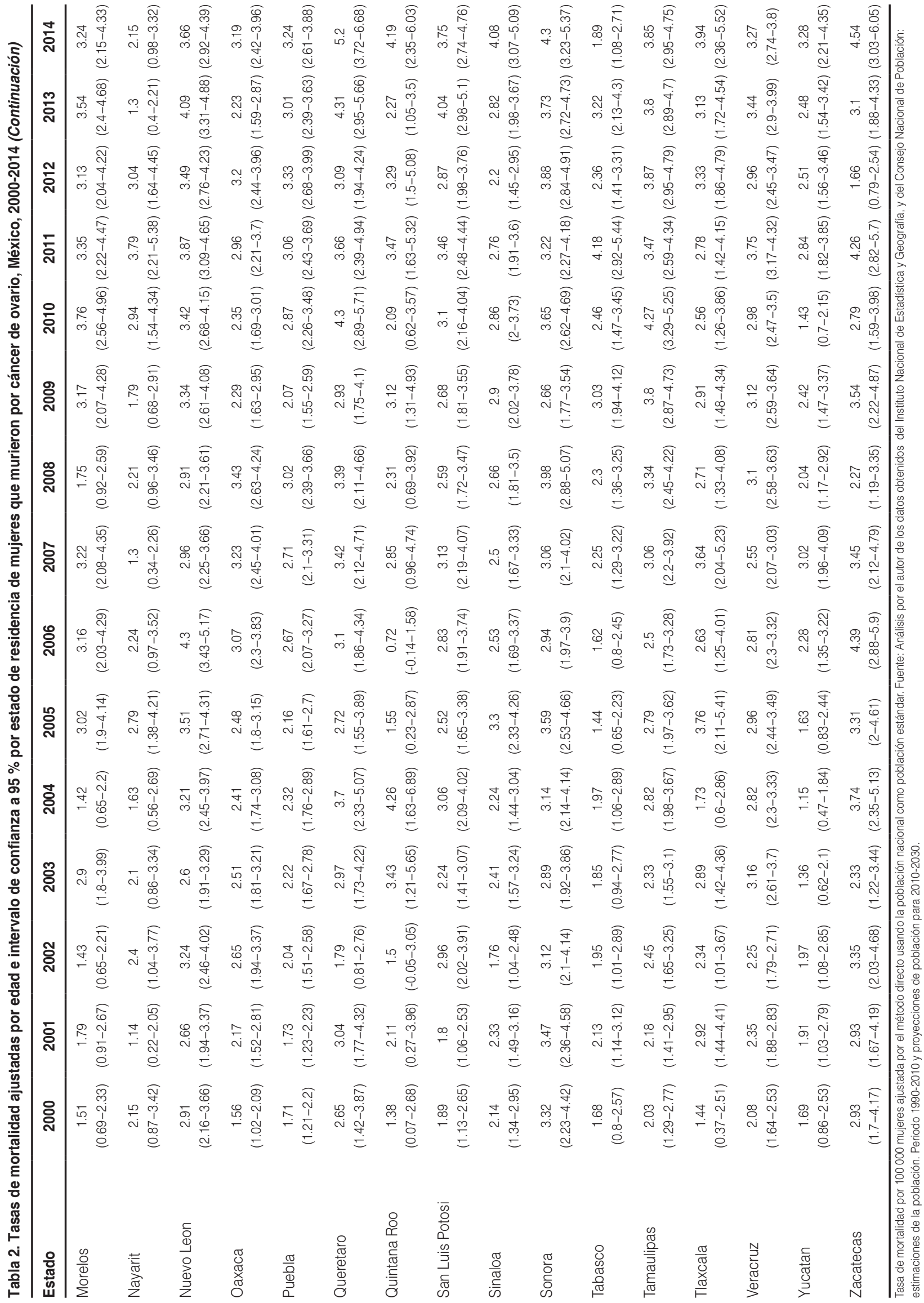




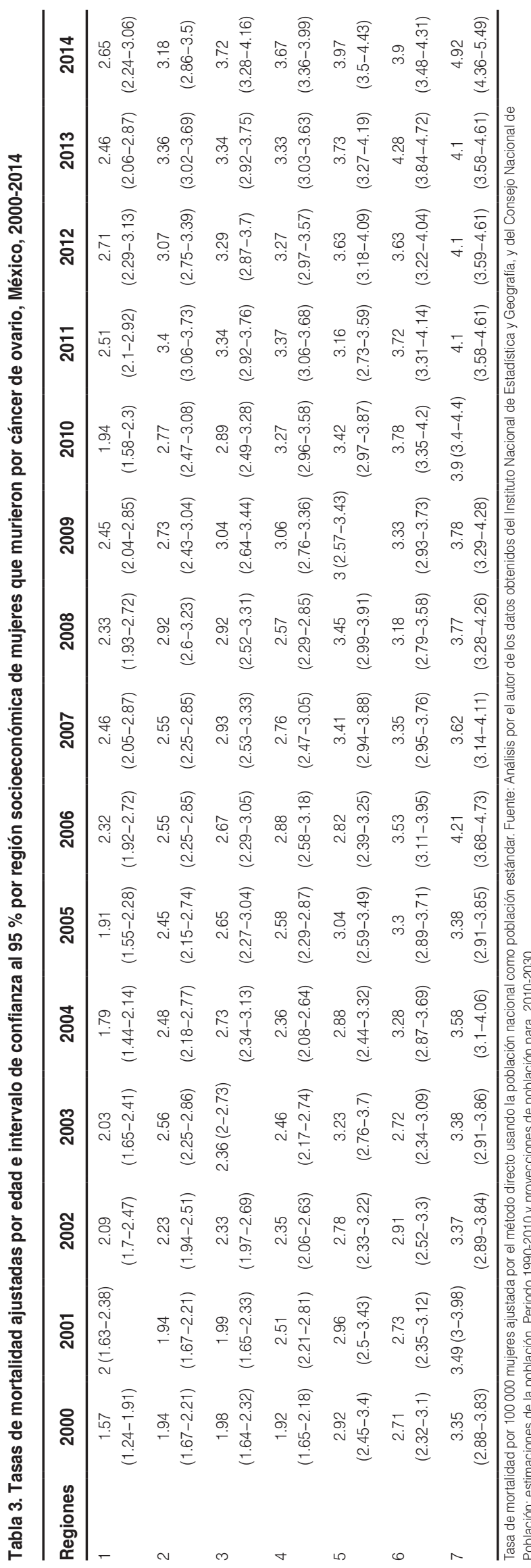

IC $95 \%=0.22-2.05 ; 1.3$, IC $95 \%=0.34-2.26 ; 1.79$, IC $95 \%=0.68-2.91 ; 1.3$, IC $95 \%=0.4-2.21$, respectivamente), Yucatán 2003 (1.36, IC $95 \%=0.62-2.1$; Guerrero en 2004 (1.03, IC $95 \%=0.54-1.52)$; Tabasco en 2005 y $2014(1.44$, IC $95 \%=0.65-2.23 ; 1.89$, IC $95 \%=1.08-2.71$, respectivamente); Quintana Roo en 2006 (0.72, IC $95 \%=-0.14-1.58)$; Campeche en 2008 (1.28, IC $95 \%=0.15-2.4)$, Chiapas en 2010 (1.36, IC $95 \%=0.86-1.86)$; Baja California Sur en 2011(1.68, IC $95 \%=0.2-3.15)$ y Zacatecas 2012 (1.66, IC $95 \%=0.79-2.54$ ) (Tabla 2).

La región socioeconómica que presentó la mayor tasa de mortalidad por $\mathrm{CO}$ en el periodo 2000-2014 fue la región 7, excepto en 2013, en el cual la región 6 presentó la mayor mortalidad. En 2000 y 2014, las tasas de mortalidad para la región 7 fueron de 3.35 , IC $95 \%=2.88-3.83$ y 4.92 , IC $95 \%=4.36-5.49$, respectivamente; para la región 6, en 2013 la tasa de mortalidad fue de 4.28, IC $95 \%=3.84-4.72$ (Tabla 3).

La región socioeconómica 1 presentó la menor tasa de mortalidad por $\mathrm{CO}$ en el periodo de estudio, excepto en 2001, en el cual la región 2 presentó la menor tasa de mortalidad. Las tasas de mortalidad para la región 1 en 2000 y 2014 fueron 1.57, IC $95 \%$ =1.241.91 y 2.65 , IC $95 \%=2.24-3.06$, respectivamente; para la región 2, en 2001 fue de 1.94, IC $95 \%=1.67$ 2.21 (Tabla 3).

Los estados con la mayor tasa de APVP en el periodo de estudio fueron Nayarit en el año 2000 (41.6), Baja California Sur en los años 2001 y 2007 (86.2 y 71.2, respectivamente), Zacatecas en 2002 y 2006 (57.5 y 76.7, respectivamente), Colima en 2003, 2004 y 2010 (81.6, 74.1 y 70.4, respectivamente), Tlaxcala en 2005 (79.6), Oaxaca en 2008 (54.4), Quintana Roo en 2009 (54.5), Coahuila en 2011 y 2012 (64 y 63.8, respectivamente), Aguascalientes en 2013 (77.8) y Querétaro en 2014 (77.4) (datos no mostrados).

Los estados con la menor tasa de APVP en el período de estudio fueron Yucatán en 2000 y 2003 (19 y 15.9, respectivamente), Guerrero en 2001 y 2014 (15.1 y 26.3, respectivamente), Quintana Roo en 2002 y 2006 (11 y 11.2, respectivamente), Nayarit en 2004, 2009 y 2013 (7.2, 27 y 6.1, respectivamente), Tabasco en 2005 (16.9), Campeche en 2007 y 2010 (18.4 y 15, respectivamente), Chiapas en 2008 (20.7), Aguascalientes en 2011 (23.3) y Zacatecas en 2012 (13.8).

Las regiones que tuvieron la mayor tasa de APVP en el periodo de estudio fueron la región 7 (en los años 2000-2004, 2006-2012 y 2014, respectivamente) y las regiones 5 (2005) y 6 (2013). Los APVP para la región 7 en 2000 y 2014 fueron 37.9 y 67.4, 
respectivamente; para la región 5 en 2005 fue de 41.4 y para la región 6 en 2013 fue de 54.6.

Las regiones que tuvieron la menor tasa de APVP en el periodo de estudio fueron las regiones 1 (en $2000,2001,2004,2005,2008$ y 2010-2014, respectivamente), la región 5 (en 2002, 2006, 2007 y 2009, respectivamente) y la región 4 (2003). Los APVP para la región 1 fueron en el año 2000 y 2014 de 22.4 y 37.5 , respectivamente; para la región 5 en 2002 y 2009 fueron de 30 y 35.5, respectivamente; y para la región 4 de 32.3 .

\section{Discusión}

En el periodo de estudio, la tasa de mortalidad por CO estandarizada por edad con la población mundial por 100000 mujeres se incrementó de 3.3 a 4.1, con un porcentaje de cambio de $24.2 \%$ y un cambio anual medio de 0.043, IC $95 \%=0.32-0.55$ (Figura 1). En el mundo, en las dos últimas décadas, las tasas de mortalidad por $\mathrm{CO}$ se han estabilizado o disminuido. Sin embargo, existen diferencias persistentes y sustanciales en los patrones y tendencias del $\mathrm{CO}^{2} \mathrm{En}$ México y en otros países, el incremento sostenido de las tasas de mortalidad por este cáncer se podría deber al diagnóstico tardío, la ausencia de quimioterapia altamente curativa y al alto grado de heterogeneidad molecular en tumores ováricos, hallazgo consecuencia de la gran carga tumoral típica en la mayoría de las pacientes al momento de la presentación de este cáncer. ${ }^{15}$

En países de altos ingresos de América del norte y Europa se ha observado que uno de los factores que ha contribuido a la disminución del $\mathrm{CO}$ es el uso extendido de los contraceptivos orales. ${ }^{2}$ El uso de anticonceptivos orales por las mujeres está asociado con una reducción del riesgo de $\mathrm{CO}$ de 40 a $50 \%$. $^{16}$ En México no hay información relacionada con el uso de contraceptivos orales y su impacto en el CO.

En este estudio se identificó que la mayor mortalidad por $\mathrm{CO}$ se presentó en el grupo de 55 a 59 años (Figura 2). Se ha observado que el riesgo de padecer $\mathrm{CO}$ se incrementa con la edad. ${ }^{1}$ Los factores que podrían estar relacionados con la presentación del $\mathrm{CO}$ al incrementarse la edad son la ovulación incesante, la estimulación por gonadotropinas y las mutaciones. Se ha observado que los ovarios de las mujeres mayores muestran más cambios morfológicos que los de las mujeres más jóvenes. El envejecimiento ovárico se caracteriza por cambios morfológicos en los ovarios como la papilomatosis, invaginaciones del epitelio superficial del ovario, quistes de inclusión y estratificaciones epiteliales, estas alteraciones podrían ser lesiones preneoplásicas que se encuentran con mayor frecuencia en mujeres posmenopáusicas y perimenopáusicas. Durante la ovulación se puede producir daño ovárico y en la posovulación hay proliferación celular, que se produce para reparar el epitelio de superficie del ovario, estos mecanismos pueden generar mutaciones que se acumulan en las células epiteliales y, finalmente, participan en la formación de tumores. La teoría de la estimulación por gonadotropinas postula que las gonadotropinas inician cada ovulación y persisten en niveles altos durante muchos años aún después de la menopausia. Las gonadotropinas estimulan a las células epiteliales superficiales ováricas e inducen la transformación celular. ${ }^{17}$

Otro factor relacionado con el incremento del CO por el incremento de la edad son las mutaciones. Los estudios genómicos indican que aproximadamente 10 a $12 \%$ de los carcinomas de ovario se caracterizan por mutaciones en los genes BRCA1 y BRCA2:15 estas mutaciones ocurren en una edad avanzada, alcanzando su máxima frecuencia en el grupo de 50 a 59 años. ${ }^{18}$

La región socioeconómica 7, que corresponde a la Ciudad de México, presentó la mayor tasa de mortalidad por CO en el periodo 2000-2014. Se ha reportado que el antecedente familiar de cáncer de ovario, la menarca temprana, la menopausia tardía, la nuliparidad, la infertilidad, el síndrome de ovario poliquístico, la terapia de reemplazo hormonal en mujeres posmenopáusicas, la exposición al asbesto, el polvo del talco, la endometriosis, la enfermedad inflamatoria pélvica, la obesidad, el consumo alto de grasas saturadas, el sedentarismo y el tabaquismo (incrementa $50 \%$ las posibilidades de $\mathrm{CO}$ mucinoso) son factores de riesgo para padecer $\mathrm{CO} .^{19}$ Algunos factores de riesgo que pudieran estar asociados con la mortalidad por cáncer de ovario en la Ciudad de México son la obesidad, el consumo alto de grasas saturadas, el sedentarismo y el tabaquismo. En la Encuesta Nacional de Salud y Nutrición se observó que la Ciudad de México tiene una de las prevalencias más altas de sobrepeso y obesidad en el país, ${ }^{20}$ las mujeres presentaron una mayor prevalencia de sobrepeso (IMC $\geq 25 \mathrm{~kg} / \mathrm{m}^{2}$ ), que los hombres (IMC $29.5 \mathrm{~kg} / \mathrm{m}^{2}$ versus $27.9 \mathrm{~kg} / \mathrm{m}^{2}$, respectivamente). La prevalencia de obesidad (IMC $\geq 30 \mathrm{~kg} / \mathrm{m}^{2}$ ) también fue mayor en las mujeres que los hombres (41\% versus $27 \%$, respectivamente, $\mathrm{p}<0.00001) .{ }^{21}$ En la Ciudad de México también se observa mayor prevalencia de inactividad física que en el resto del país $(41.4 \%) .{ }^{20}$ La inactividad física en 


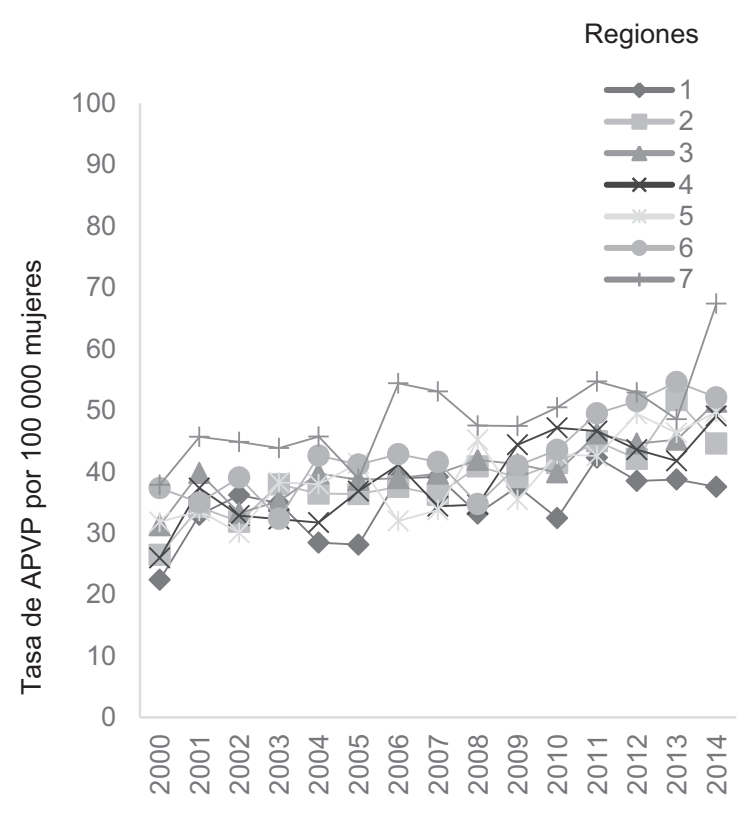

Año de muerte

Figura 3. Tasa de años potenciales de vida perdidos por cáncer de ovario según región socioeconómica. México, 2000-2014. Tasa de APVP por 100000 mujeres ajustada por edad usando la población nacional como población estándar de referencia.

la Ciudad de México se ha reportado en $82 \%$ en mujeres y $71 \%$ en hombres. ${ }^{21}$ La Ciudad de México tiene una de las más altas prevalencias de tabaquismo en el país. La Encuesta de Tabaquismo en Jóvenes en México ${ }^{22}$ reportó que la Ciudad de México tuvo la mayor prevalencia de jóvenes fumadores (27.8\%); $58.3 \%$ de las mujeres tenía hábito tabáquico.

La región 7 es la región con las mejores condiciones socioeconómicas del país; en el periodo de estudio presentó las mayores tasas de APVP (Figura 3) y de mortalidad por CO (Tabla 3).

Los APVP son una de las medidas del impacto relativo de varias enfermedades y problemas de salud en la sociedad, que ilustran sobre las pérdidas que sufre la sociedad como consecuencia de la muerte de personas jóvenes o de fallecimientos prematuros. Se considera que una muerte es prematura cuando ocurre antes de cierta edad predeterminada, que corresponde por ejemplo a la esperanza de vida al nacer en la población estudiada. Considerar la edad a la cual mueren las personas y no sólo el evento mismo de la muerte permite asignar un peso diferente a las muertes que ocurren en diferentes momentos de la vida. El supuesto en el que se basan los APVP es que cuando más "prematura" es la muerte, mayor es la pérdida de vida, algunas de las cuales son sensibles a las medidas sanitarias y sociales. ${ }^{12}$
La Sociedad Americana de Ginecología y Cáncer recomienda las siguientes estrategias para la prevención del CO:16

- Uso de anticonceptivos orales.

- Oclusión tubaria.

- Reducción de riesgo mediante salpingo-ooforectomía en mujeres con alto riesgo hereditario de cáncer de mama y ovario,

- Asesoramiento genético y pruebas de genética para mujeres con alto riesgo familiar de $\mathrm{CO}$.

- Salpingectomía al tener la paridad satisfecha.

\section{Conclusiones}

En el periodo de estudio 25047 mujeres murieron por $\mathrm{CO}$, la tasa de mortalidad estandarizada por edad con la población mundial por 100,000 mujeres se incrementó de 3.3 a 4.1, con un porcentaje de cambio de $24.2 \%$ y un cambio anual medio de 0.043, IC $95 \%=0.32-0.55$. En el periodo de estudio, los estados y la región socioeconómica que presentaron las mayores tasas de mortalidad fueron Chihuahua (2000), Baja California Sur (2001, 2002, 2007 y 2009), Colima (2003, 2005, 2011, 2012), Quintana Roo (2004), Zacatecas (2006), Sonora (2008), Coahuila (2010), Aguascalientes (2013), Querétaro (2014) y la región 7 . Los estados y las regiones que presentaron la mayor tasa de APVP en el periodo de estudio fueron Nayarit (2000), Baja California Sur (2001 y 2007), Zacatecas (2002), Colima (2003, 2004 y 2010), Tlaxcala (2005), Zacatecas (2006), Oaxaca (2008), Quintana Roo (2009), Coahuila (2011-2012), Querétaro (2014) y las regiones 7 (2000-2004, 2006-2012, 2014), 5 (2005) y 6 (2013).

\section{Bibliografía}

1. Ovarian Cancer 2014 Report. Food, nutrition, physical activity, and the prevention of ovarian cancer. Reino Unido: World Cancer Research Fund/American Institute for Cancer Research; 2014. Disponible en: www. wcrf.org/sites/default/files/Ovarian-Cancer-2014-Report.pdf

2. Malvezzi M, Carioli G, Rodríguez T, Negri E, La-Vecchia C. Global trends and predictions in ovarian cancer mortality. Ann Oncol. 2016;27:2017-2025.

3. Rivas-Corchado LM, González-Geroniz M, Hernández-Herrera RJ. Epidemiological profile of ovarian cancer. Ginecol Obstet Mex. 2011; 79:558-564.

4. Torres-Sánchez LE, Rojas-Martínez R, Escamilla-Núñez C Vara-Salazar E, Lazcano-Ponce E. Tendencias en la mortalidad por cáncer en México de 1980 a 2011. Salud Publica Mex. 2014;56:473-491.

5. Instituto Nacional de Estadística y Geografía. Registros administrativos de mortalidad. México: Instituto Nacional de Estadistica y Geografía; 2016. Disponible en: http://www3.inegi.org.mx/sistemas/microdatos/encuestas.aspx?c=33398\&s=est

6. World Health Organization. International Statistical Classification of Diseases and Problems Related to Health, $10^{\text {th }}$ review. Ginebra, Suiza: World Health Organization; 1995.

7. Inskip H, Beral V, Fraser P, Haskey J. Methods for age-adjustment of rates. Stat Med. 1983;2:455-466.

8. Ahmad OB, Boschi-Pinto C, López AD, Murray CJL, Lozano R, Inoue M. Age standardization of rates: a new WHO standard. En: Global Program- 
me on Evidence for Health Policy Discussion Paper Series no. 31. Ginebra, Suiza: World Health Organization; 1999.

9. Instituto Nacional de Estadistica y Geografía. Regiones socioeconómicas de México México: Instituto Nacional de Estadistica y Geografía; 2000 Disponible en :http://sc.inegi.org.mx/niveles/datosnbi/reg_soc_mexico. pdf

10. Consejo Nacional de Población. Estimaciones demográficas 1990-2010 y proyecciones de la población 2010-2050. México: Consejo Nacional de Población; 2014. Disponible en. http://www.conapo.gob.mx/es/CONAPO/Proyecciones_Datos.

11. Jensen OM, Parkin DM, MacLennan R, Muir CS, Skeet RG. Cancer registration: principles and methods. Francia: International Agency for Research on Cancer; 1995.

12. Pan American Health Organization. Techniques to measure the impact of mortality: years of potential life lost. Epidemiological Bulletin. 2003; 24:1-4.

13. Romeder JM, McWhinnie JR. Potential years of life lost between ages 1 and 70: an indicator of premature mortality for health planning. Int $\mathrm{J}$ Epidemiol. 1977:6:143-151.

14. Hervada-Vidal X, Santiago-Pérez M, Vázquez-Fernández E, Castillo-Salgado C, Loyola-Eñizondo E, Silva-Ayçaguer LC. Epidat 3.0 Programa para análisis epidemiológico de datos tabulados. Rev Esp Salud Publica. 2004;78:277-280.

15. Hunn J, Rodríguez GC. Ovarian cancer: etiology, risk factors, and epidemiology. Clin Obstet Gynecol. 2012;55:3-23.
16. Walker JL, Powell CB, Chen LM, Carter J, Bae-Jump VL, Parker LP, et al. Society of Gynecologic Oncology recommendations for the prevention of ovarian cancer. Cancer. 2015;121:2108-2120.

17. Smith ER, Xu XX. Ovarian ageing, follicle depletion, and cancer: a hypothesis for the aetiology of epithelial ovarian cancer involving follicle depletion. Lancet Oncol. 2008;9:1108-1111.

18. Song H, Cicek MS, Dicks E, Harrington P, Ramus SJ, Fridley BL, et al. The contribution of deleterious germline mutations in BRCA1, BRCA2 and the mismatch repair genes to ovarian cancer in the population. Hum Mol Genet. 2014;23:4703-4709.

19. Reid BM, Permuth JB, Sellers TA. Epidemiology of ovarian cancer: a review. Cancer Biol Med. 2017:14:9-32.

20. Encuesta Nacional de Salud y Nutrición de Medio Camino 2016. Informe final de resultados. México: Secretaría de Salud/Instituto Nacional de Salud Pública; 2016.

21. Kuri-Morales P, Emberson J, Alegre-Díaz J, Tapia-Conyer R, Collins R, Peto $R$, et al. The prevalence of chronic diseases and major disease risk factors at different ages among 150000 men and women living in Mexico City: cross-sectional analyses of a prospective study. BMC Public Health. 2009;9:9.

22. Reynales-Shigematsu L, Valdés-Salgado R, Rodríguez-Bolaños R, Lazcano-Ponce E. Hernández-Ávila M. Encuesta de tabaquismo en jóvenes en México. Análisis descriptivo 2003, 2005, 2006, 2008. México: Instituto Nacional de Salud Pública/Secreataría de Salud/Comisión Nacional contra las Adicciones; 2009 